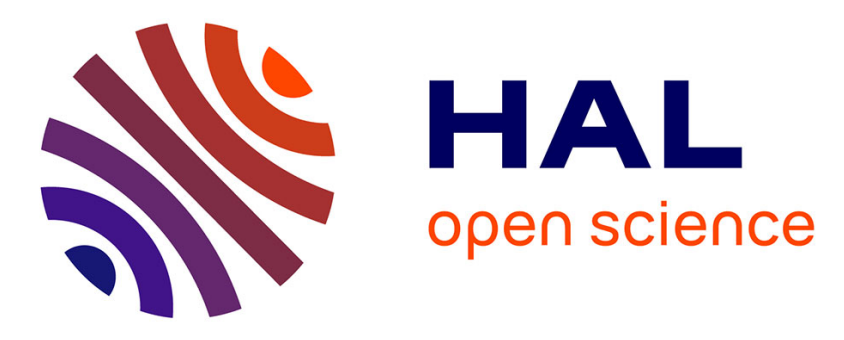

\title{
Assessing tumour vascularity with 3D contrast-enhanced ultrasound; a new semi-automated segmentation framework
}

\author{
Anne Gasnier, Roberto Ardon, Cybèle Ciofolo-Veit, Edward Leen, \\ Jean-Michel Corréas
}

\section{To cite this version:}

Anne Gasnier, Roberto Ardon, Cybèle Ciofolo-Veit, Edward Leen, Jean-Michel Corréas. Assessing tumour vascularity with 3D contrast-enhanced ultrasound; a new semi-automated segmentation framework. 2010 IEEE International Symposium on Biomedical Imaging: From Nano to Macro, Apr 2010, Rotterdam, Netherlands. pp.300-303, 10.1109/ISBI.2010.5490351 . hal-00508785

\section{HAL Id: hal-00508785 \\ https://hal.science/hal-00508785}

Submitted on 5 Aug 2010

HAL is a multi-disciplinary open access archive for the deposit and dissemination of scientific research documents, whether they are published or not. The documents may come from teaching and research institutions in France or abroad, or from public or private research centers.
L'archive ouverte pluridisciplinaire HAL, est destinée au dépôt et à la diffusion de documents scientifiques de niveau recherche, publiés ou non, émanant des établissements d'enseignement et de recherche français ou étrangers, des laboratoires publics ou privés. 


\title{
ASSESSING TUMOUR VASCULARITY WITH 3D CONTRAST-ENHANCED ULTRASOUND: A NEW SEMI-AUTOMATED SEGMENTATION FRAMEWORK
}

\author{
A. Gasnier ${ }^{1,2}$, R. Ardon ${ }^{1}$, C. Ciofolo-Veit ${ }^{1}$, E. Leen ${ }^{2}$, and J.M. Correas ${ }^{3}$ \\ ${ }^{1}$ Philips Medical Systems Research Paris, Suresnes, France \\ ${ }^{2}$ Imaging Sciences Department, Imperial College, London, UK \\ ${ }^{3}$ Department of Adult Radiology, Necker Hospital, Paris, France
}

\begin{abstract}
3D contrast-enhanced ultrasound (CEUS) is a powerful imaging technique for tumour vascularity assessment, which is critical for radio-frequency ablation (RFA) planning or for the assessment of response to antiangiogenic therapies. In this paper, we propose a novel semi-automated method for the quantification of tumour vascularity in 3D CEUS data. We apply a two-step framework combining an interactive segmentation of the tumour necrosis followed by an automatic detection of the vascularity based on implicit representations. Experimental results on 3D CEUS images of renal cell carcinomas (RCC) show that our method is promising in terms of speed and quality.
\end{abstract}

Index Terms - Tumour Vascularity Segmentation, 3D Contrast Enhanced Ultrasound.

\section{INTRODUCTION}

Recent advances in ultrasound imaging techniques have led to a marked improvement in contrast-enhanced volumetric imaging. 3D ultrasound offers a better knowledge of heterogeneous objects than 2D ultrasound. Ultrasound contrast agents (UCA) increase the amplitude of the scattered signal from the micro-vasculature, enabling tissue perfusion imaging [1]. Though UCA are not approved for radiology imaging in the United States at this time, they are popular and widely used in Europe and Asia. Fig.1 shows that UCA enhance the visualization of tumour vascularity. As UCA don't diffuse in the interstitial space contrary to MRI or CT contrast agents, they are well suited for the quantification of tumour vasculature volume. The use of quantitative imaging for the characterization of tumours can improve the diagnosis, the planning and the monitoring of tumour treatments, specifically in the clinical contexts of RFA and antiangiogenic therapies [2].

RFA is a minimally invasive therapy of tumours for patients who do not meet the criteria for surgical resectability. The aim of thermal ablation is to produce coagulation necrosis by using cytotoxic heat. An accurate delineation of tumour vascularity in a 3D image improves the planning of the needle insertion as well as the monitoring of the treatment
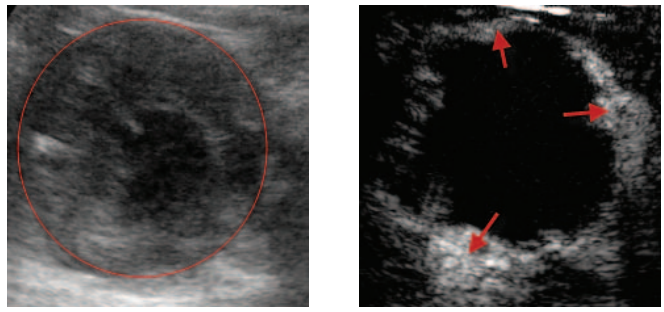

Fig. 1. Left: ultrasound B mode image of a kidney tumour (circle). Right: ultrasound harmonic imaging of this tumour, its vascularity being enhanced by ultrasound contrast agents (arrows).

outcome by comparing the overlap between the pre-ablation tumoural volume and the post-ablation necrosis. Antiangiogenic drugs slow down the progression of a cancer by inhibiting the angiogenesis, i.e. preventing the development of tumoural blood vessels. Monitoring the tumour vascularity over time is of paramount importance to evaluate the patient response to treatment. It is of great interest to delineate accurately a volume of interest in which the tumour vascularity kinetic can be studied through the injection of UCA.

In current practice, measurements on 3D CEUS images of tumours are mainly achieved through tedious and timeconsuming manual delineations or through the use of simple shapes, such as ellipsoids, that poorly account for tumour heterogeneity. While extensive work has been performed on tumour segmentation algorithms, to our knowledge, no solution for tumour vascularity segmentation on 3D CEUS images have been proposed yet [3].

We propose a novel computer-assisted tool that performs 3D segmentations of both tumour necrosis (section 2.1) and vascularity (section 2.2). Necrosis segmentation is obtained from an adaptation of an interactive algorithm combining image features and the radiologist's clinical knowledge [4]. It copes efficiently with poor image quality and high necrosis shape variability. Tumour vascularity is then segmented automatically using a shape-constrained approach based on its ring-like shape on the periphery of the necrosis. In section 3 we compare our algorithm to manual segmentations performed by a radiologist. 


\section{MATERIALS \& METHODS}

\subsection{Tumour necrosis segmentation}
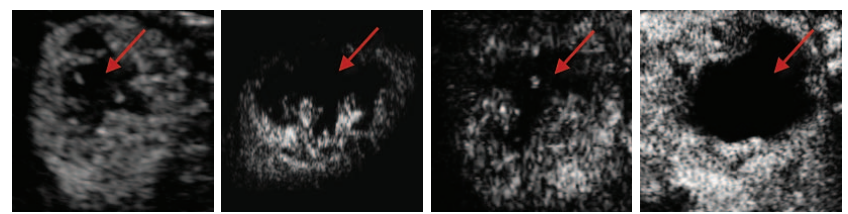

Fig. 2. RCC tumours present a range of necroses shapes (arrows).

In addition to patient-dependent image quality (e.g: misleading anechoic regions), tumour necroses present a high variability in shape (see Fig.2). Manual segmentation of necrosis requires a high level of expertise and stems from the combination of expert's knowledge and image features. The interactive algorithm introduced in [4] was specifically designed to allow rapid 3D segmentations, optimally combining user interactions and image information. User interactions are as simple as clicks on the image, specifying whether a point in the image is inside or outside the necrosis. In practice few interactions (less than 5 points) are needed to obtain satisfactory segmentations without tedious delineations. Typically a first click inside the necrosis initiates a fairly accurate segmentation but "leakages" are common due to missing edge information. A few additional clicks around "leakages" or "missing edges" produce satisfactory corrections. Fig.3 illustrates the process on a synthetic 3D image.

This method is based on an implicit representation of the necrosis region as a linear combination of image dependent kernels (geodesic radial basis functions). The algorithm sequentially builds this representation as the user introduces points in the image. At the introduction of the $n^{\text {th }}$ point $x_{n}$, the algorithm computes ([4]) an apodized geodesic distance $x \rightarrow \varphi\left(\left\|x-x_{n}\right\|_{g_{n}}\right)$ where $g_{n}$ is a metric function and $\varphi$ is a decreasing non-negative kernel.The necrosis segmentation is then obtained by the zero super-level $(\{x, \Phi(x) \geq 0\})$ of a linear combination of the previous interactions and the $n^{\text {th }}$ apodized geodesic distance:

$$
\Phi_{\lambda_{i=1 . . n}}(x)=\sum_{i=1}^{n} \lambda_{i} \varphi\left(\left\|x-x_{i}\right\|_{g_{i}}\right) .
$$

The $n$ coefficients $\left\{\lambda_{i}\right\}_{i=1 . . n}$ are obtained by solving the constrained optimization problem:

$$
\min _{\lambda_{1}, \ldots, \lambda_{n}} E\left(\Phi_{\lambda_{i}}\right)=\int_{\Omega} H\left(\Phi_{\lambda_{i}}\right) r_{a}(I)+\int_{\Omega}\left(1-H\left(\Phi_{\lambda_{i}}\right)\right) r_{b}(I)
$$

where $I$ is the image intensity, $H$ is the Heaviside function, $r_{a}(I)=-\log P_{\Omega_{a}}(I), r_{b}(I)=-\log P_{\Omega \backslash \Omega_{a}}(I)$, and $P_{\Omega_{a}}, P_{\Omega_{b}}$ are pixels intensities within the necrosis and the exterior respectively. This problem is minimized under a set of linear constraints specified as the user imposes a control point to be inside $\left(\Phi\left(x_{n}\right) \geq 0\right)$ or outside the necrosis $\left(\Phi\left(x_{n}\right)<0\right)$.

The adaptation of this algorithm to our problem is done by choosing a specific distance metric. Necroses are generally characterized by homogeneous image intensity and high image gradient on its boundary. The following metric: $g_{n}(x)=$ $\left(I(x)-I\left(x_{n}\right)\right)^{2} \times|\nabla I|^{2}$ accounts for these features.
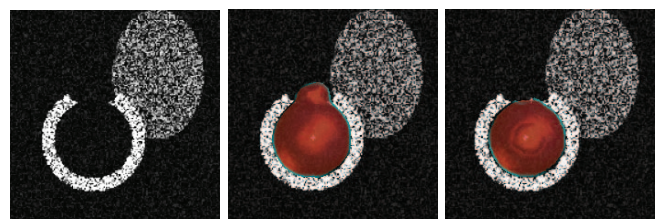

Fig. 3. Necrosis segmentation on a synthetic image. From left to right: a synthetic image; the resulting segmentation after one click; the final segmentation after one correction.

\subsection{Vascularity segmentation}

Through angiogenesis, a tumour develops new vascular structures in order to reach more nutrients and oxygen. As tumour size increases, necrosis develops in its center where cells are remote from blood supply. Therefore tumour vascularity often adopts a ring-like distribution on the periphery of the necrosis.

The use of ultrasound contrast agents enhances both the vascularity/necrosis and vascularity/surrounding tissue interfaces. The former presents a strong image gradient (Fig.1), the latter presents image texture differences (Fig.4). Indeed RCC are generally less well perfused than the cortex [5].

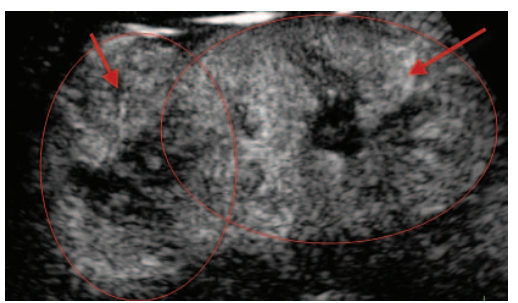

Fig. 4. The tumour (oval on the left side of the image) is located partly in the kidney (oval on the right side). Both the tumour and the kidney are vascularized and enhanced by UCA (arrows).

Based on these image features, we propose a minimization algorithm for the automatic segmentation of tumour vascularity. The functional to be minimized $(E)$ is composed of four different terms rationalizing the vascularity ring-like shape $\left(E_{c}\right)$, high image gradient at the interface with necrosis $\left(E_{e}\right)$, intensity dissimilarity measure with surrounding tissue $\left(E_{i}\right)$ and a regularization term $\left(E_{r}\right)$. Mathematically, we represent the vascularity as the zero level set of a bounded variations function $u$ restricted to $[-1 ; 1]$, partitioning the image domain $\Omega$ in two regions $\Omega_{\mathrm{int}}=\{x \in \Omega, u(x) \geq 0\}$ and $\Omega_{\mathrm{ext}}=\{x \in \Omega, u(x)<0\}$ (Fig.5). The expression of the functional we optimize is

$$
\min _{u \in B V_{[-1,1]}} E(u)=\underbrace{E_{i}(u)+E_{e}(u)}_{\text {image-based }}+\underbrace{E_{c}(u)}_{\text {constraint }}+\underbrace{E_{r}(u)}_{\text {regularization }}
$$



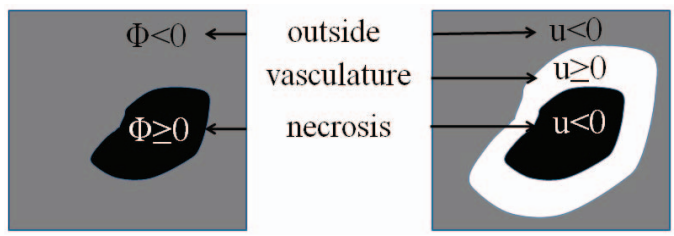

Fig. 5. Left: implicit function for the necrosis segmentation. Right: implicit function for the vasculature segmentation.

We now give the expressions of each of these terms.

Intensity distributions dissimilarity measure: As shown in [6], a suitable metric to quantify the similarity between two regions is given by the Wasserstein distance between image intensity distributions. Within our formalism, the Wasserstein distance between the image intensity distributions inside $(u \geq$ $0)$ and outside $(u<0)$ the vascularity is :

$$
W(u)=\int_{\mathbb{R}}\left|F\left(y, \frac{1+u}{2}\right)-F\left(y, \frac{1-u}{2}\right)\right| d y
$$

where $F$ is the cumulative distribution function defined by:

$$
F(y, v)=\left(\int_{\Omega} v(x) d x\right)^{-1} \int_{a=0}^{y} \int_{\Omega} \delta(I(x)-a) v(x) d x d a
$$

$\delta$ being the Dirac distribution. We choose the expression $E_{i}(u)=-W(u)$, as minimizing $E_{i}$ increases the intensity distributions distance between the regions defined by $u$.

Image gradient: As the vascularity is enhanced, the image gradient at its interface with the necrosis or the surrounding tissue is oriented toward its interior. Our gradient term must favor an interface $\{u=0\}$ minimizing the outward gradient flow:

$$
E_{e}(u)=\int_{\{u=0\}} \nabla \vec{I} \cdot \vec{n}
$$

where $\vec{n}$ is the outward unit normal of the surface $\{u=0\}$. Ring-like shape constraint: Segmentations that differ from a ring-like shape are constrained through the following term:

$$
E_{c}(u)=\int_{\Omega}(u(x)-S(x))^{2} d x
$$

where $S$ is a discrete function satisfying $S=1$ inside the ring model and $S=-1$ outside. The construction of this model is described further on.

Regularization term: Finally we regularize the segmentation by using a total variation (TV) term:

$$
E_{r}(u)=\int_{\Omega}|\nabla u(x)| d x
$$

Shape model, Implementation and Initialization: Due to the difficulty of CEUS images, we cannot apply our vascularity segmentation algorithm without restricting the image domain to a vicinity of the necrosis (previously segmented). This restriction, as well as the definition of the function $S$ and the initialization of $u$, are made through the following procedure.

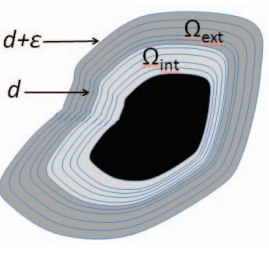

Fig. 6. Restriction of the image domain
From the distance function $D$ to the necrosis, we consider the following sets:

$$
\begin{aligned}
& \Omega_{\text {int }}(d)=\{x, D(x) \in[0 ; d]\} \\
& \Omega_{\text {ext }}(d)=\{x, D(x) \in[d ; d+\epsilon]\}
\end{aligned}
$$

where $d$ is a particular distance level and $\epsilon$ is the inverse of the necrosis radius. We define the discrete function

$$
\tilde{u}(x, d)=\left\{1 \text { if } x \in \Omega_{\text {int }}(d),-1 \text { if } x \in \Omega_{\text {ext }}(d)\right\} .
$$

We compute $E^{\prime}(d)=\left(E_{i}+E_{e}\right)(\tilde{u}(x, d))$ for a suitable set of discrete distance values and we determine a minimal value and its corresponding distance level $\tilde{d}$. With this fast and simple approach we restrict our image domain to $\Omega=\Omega_{\text {int }}(\tilde{d}) \cup$ $\Omega_{\text {ext }}(\tilde{d})$. We initialize the function $u$ with $u=\tilde{u}(., \tilde{d})$ and we define the shape constraint $S$ as $S=\tilde{u}(., \tilde{d})$.

The minimization of the functional $E$ is done using an exact penalization term [7] to restrict $u$ to $[-1,1]$ and an alternated gradient descent scheme (decoupling TV-regularization and gradient descent) as proposed in [8]. Due to space limitations, we omit the details on these algorithms and we refer the interested reader to [7, 8]. Fig.7 shows the result of the vascularity segmentation on a synthetic image (see Fig.3).
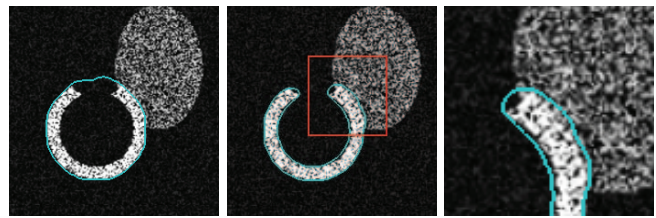

Fig. 7. Segmentation of the vasculature on a synthetic image. From left to right: level $\tilde{d}$ detected; final segmentation of the necrosis; zoom-in view of the final segmentation (box).

\section{RESULTS}

3D CEUS data were acquired on 2 phantoms and 5 patients with RCC carcinoma following the administration of $2 \mathrm{~mL}$ of the UCA SonoVue (Bracco, Milan, Italy). All 5 patients underwent CT-scans. The necrosis and the vascularity of each tumour have been manually segmented on several slices of both the CEUS and CT data, using a tool that we developed in $\mathrm{C}++$ based on the VTK library ${ }^{1}$. 3D semi-automatic quantification measurements were compared to manual segmentation of ultrasound images. Fig. 8 shows the 3D segmentation of a kidney tumour necrosis and vasculature and Fig.9 shows the result on five kidney tumours.

${ }^{1}$ www.vtk.org: VTK is an open-source, freely available software system for $3 \mathrm{D}$ computer graphics, image processing and visualization. 

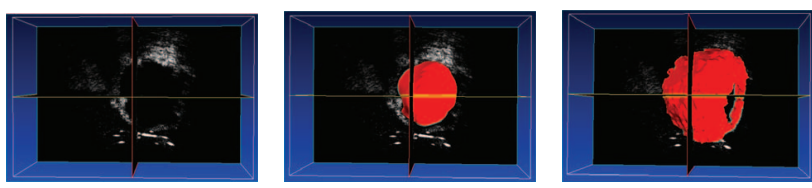

Fig. 8. From left to right: a kidney tumour; the 3D segmentation of the necrosis; the $3 \mathrm{D}$ segmentation of the vasculature.
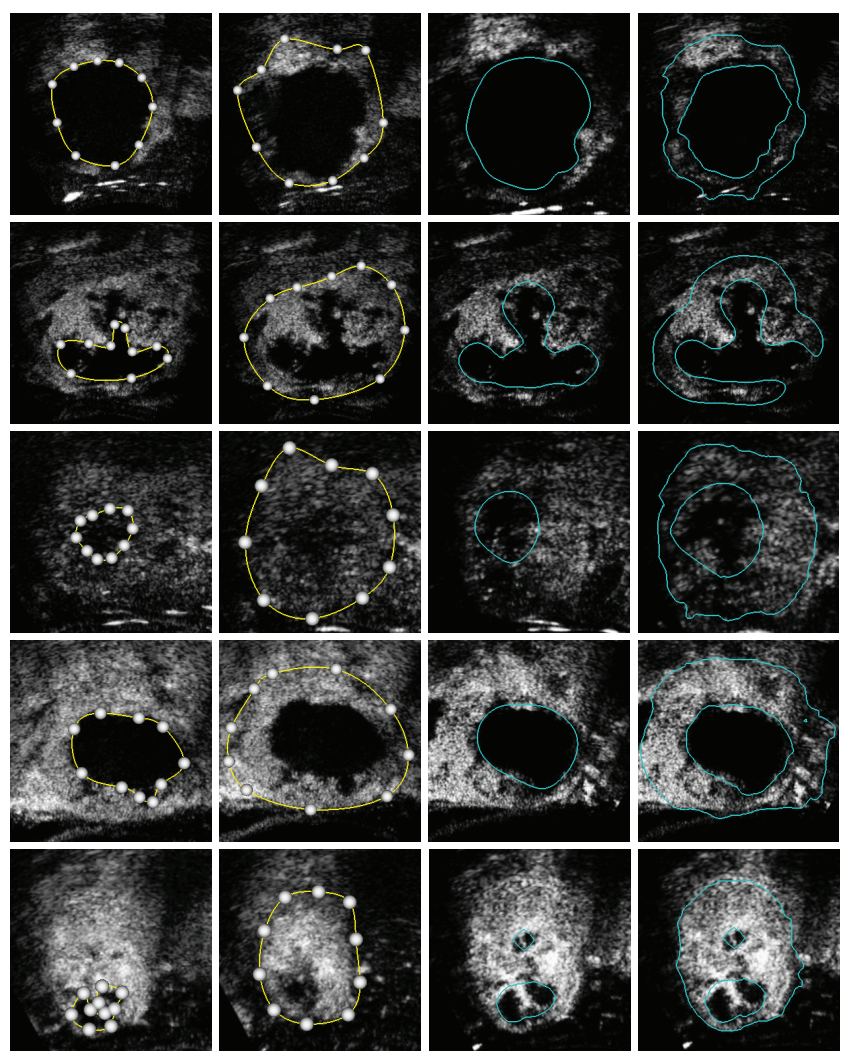

Fig. 9. Segmentation of the necrotic and vascularized volume of kidney tumours (from top to bottom). 2 images on the left: manual delineation. 2 images on the right: semi-automatic segmentations.

The average segmentation time was 1 minute for the semiautomatic segmentation and 10 minutes for the manual delineation. The average axial, lateral and elevation resolutions of CEUS data were $0.4 \mathrm{~mm}, 0.3 \mathrm{~mm}$ and $0.7 \mathrm{~mm}$. The average diameter of the tumours measured from CEUS data was $6 \mathrm{~cm}$. On phantom studies our segmentations show less than $5 \%$ error and on clinical data the average error in the delineation is $20 \%$ for both the necroses and the vasculatures. Those errors can be compared to the variation in volume from manual delineations of CEUS and CT images. The average variation in volume ranged from $14 \%$ for the necroses to $16 \%$ for the vascularized volumes. Those variations can be explained by the poor quality of CEUS images that requires clinicians to extrapolate lesion boundaries. Therefore our results are promising considering the quality of ultrasound data sets.

\section{DISCUSSION \& FUTURE WORK}

The newly developed semi-automatic tool is promising as it rapidly provides accurate measurements of tumour volumes. This technique may be useful for the evaluation of response to cytotoxics as well as antiangiogenic drugs and ablative therapies by assessing altered vascularized volume. One further improvement of the method would be the addition of interactions for the vascularity segmentation in order to provide radiologists with some flexibility for delineating the tumour vascularity. One drawback of our vascularity segmentation algorithm is its inability to control topological changes. In order to fully control the shape of the vascularity segmentation, we are working on the implementation of an interactive segmentation of the tumour vascularity by applying deformations to a ring model. Further steps include more extensive clinical validation as well as the study of the evolution over time of volume signal intensity within the vascularized region of interest.

\section{REFERENCES}

[1] T. Albrecht, M. Blomley, L. Bolondi, M. Claudon, JM Correas, D. Cosgrove, E. Leen, R. Lencioni, D. Lindsell, A. Martegani, et al., "Guidelines for the use of contrast agents in ultrasound," Ultraschall Med, vol. 25, no. 4, pp. 249-256, 2004.

[2] E. Leen, S. Kumar, S.A. Khan, G. Low, K.O. Ong, P. Tait, and M. Averkiou, "Contrast-enhanced 3d ultrasound in the radiofrequency ablation of liver tumors," WJG, vol. 15, no. 3, pp. 289, 2009.

[3] J.A. Noble and D. Boukerroui, "Ultrasound image segmentation: A survey," IEEE Transactions on medical imaging, vol. 25, no. 8, pp. 987-1010, 2006.

[4] B. Mory, R. Ardon, A.J. Yezzi, and J.P. Thiran, "Noneuclidean image-adaptive radial basis functions for $3 \mathrm{~d}$ interactive segmentation," in ICCV, 2009, pp. 787-794.

[5] D Cosgrove, "Developments in ultrasound," Imaging, vol. 18, no. 2, pp. 82-96, 2006.

[6] T. Chan, S. Esedoglu, and K. Ni, "Histogram based segmentation using wasserstein distances," Lecture Notes In Computer Science, vol. 4485, pp. 697, 2007.

[7] M. Nikolova, S. Esedoglu, and T. Chan, "Algorithms for finding global minimizers of image segmentation and denoising models," SIAM Journal on Applied Mathematics, vol. 66, no. 5, pp. 1632-1648, 2006.

[8] B. Mory and R. Ardon, "Fuzzy region competition: A convex two-phase segmentation framework," Lecture Notes in Computer Science, vol. 4485, pp. 214, 2007. 